\title{
Microgravity Vibration Isolation for the International Space Station
}

\author{
Mark S. Whorton \\ TD55Nehicle Control Systems, NASA Marshall Space Flight Center, Huntsillle, AL 35812 \\ Telephone: 256-5+4-1435; email: mark.whorton@msfc.nasa.gor
}

\begin{abstract}
The International Space Station (ISS) is being envisioned as a laboratory for experiments in numerous microgravity $(\mu \mathrm{g})$ science disciplines. Predictions of the ISS acceleration environment indicate that the ambient acceleration levels will exceed levels that can be tolerated by the science experiments. Hence, microgravity vibration isolation systems are being developed to attenuate the accelerations to acceptable levels. While passive isolation systems are beneficial in certain applications, active isolation systems are required to provide attenuation at low frequencies and to mitigate directly induced payload disturbances. To date, three active isolation systems have been successfully tested in the orbital environment. A fourth system called g-LIMIT is currently being developed for the Microgravity Science Glovebox and is manifested for launch on the UF-1 mission. This paper presents an overview of microgravity vibration isolation technology and the g-LIMIT system in particular.
\end{abstract}

\section{INTRODUCTION}

The orbital environment provides a unique opportunity for studying phenomena in a manner not possible on earth. Earth-orbiting spacecraft provide the potential for a low-level acceleration environment enabling microgravity $(\mu g)$ science experiments in disciplines such as life sciences, materials science, combustion, fundamental physics, and fluid mechanics. As a research laboratory, the International Space Station (ISS) will exploit the near-zero acceleration environment of low-earth orbit for unique state-of-the-art $\mu \mathrm{g}$ science investigations. However, due to a variety of vibro-acoustic disturbances on the ISS, the acceleration environment is expected to significantly exceed the requirements of many acceleration sensitive experiments. Figure 1 presents an estimate of the acceleration environment on the ISS along with the required acceleration levels for $\mu \mathrm{g}$ science from the ISS Microgravity Environment Specification.' Mitigation of the excessive acceleration environment requires the implementation of vibration isolation systems at either the disturbance source or the science payload. While an effort is being made to limit the induced disturbances, it is understood that the acceleration levels will not meet the environment requirement specification, thus requiring the use of vibration isolation at the payload/rack locations.

In view of the utility of the ISS as an orbiting science laboratory, the need for vibration isolation systems for acceleration sensitive experiments is gaining increasing visibility. To date, three active vibration isolation systems have been flight tested on shuttle flights. The STABLE (Suppression of Transient Accelerations By Levitation) system, developed jointly by NASA Marshall Space Flight Center (MSFC) and The Boeing Corporation (formerly McDonnell Douglas Aerospace Corporation), was the first successful orbital flight test of an active $\mu \mathrm{g}$ vibration isolation system." Flown on the STS-73/USML-02 shuttle flight in October 1995, STABLE was integrated in a modified Middeck Locker and isolated a fluid dynamics experiment using non-contact electromagnetic Lorentz force actuators and a high gain acceleration feedback control system.

The second system flight tested was the Microgravity Isolation Mount (MIM) developed by the Canadian Space Agency. ${ }^{3}$ The MIM device is also a component-level acceleration feedback based active isolation system. The first MIM unit was developed and launched by CSA as a NASA payload on the Priroda laboratory module, which docked with the Russian Mir space station in April 1996. The first MIM system began operating on the Mir in May 1996. accumulating more than 1700 hours of operation supporting several fluid physics experiments. An upgraded system (MIM-2) was flown on STS-85 in August 1997. The MIM system requires one Middeck locker volume for the isolation system, which interfaces to an experiment through a tabletop interface. 


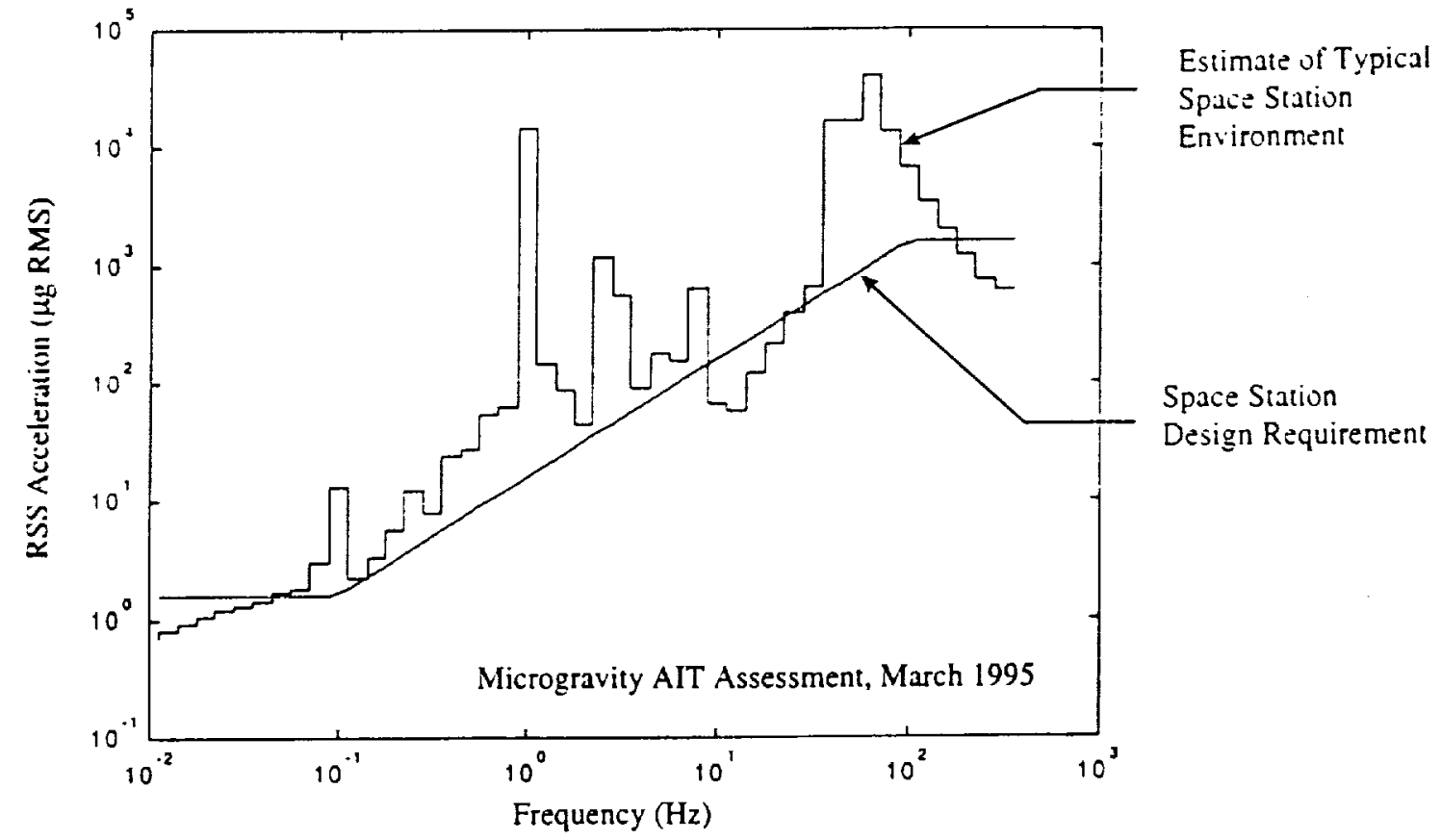

FIGURE 1. Predicted Acceleration Environment of ISS

The Boeing Active Rack Isolation System (ARIS) is the third system to be successfully flight tested on the space shuttle. ${ }^{4}$ The basic ARIS concept focused on providing isolation for an entire International Standard Payload Rack (ISPR) at the rack to ISS structural interface. NASA base-lined ARIS in order to provide ISS with an acceleration environment as defined in Figure 1 at a fixed number of rack locations. Of the three systems, ARIS is the only racklevel isolation system. While all three systems use electromagnetic Lorentz force actuators, the ARIS racks utilize voice coil rotary actuators and pushrods to connect the rack and station structure. The ARIS Risk Mitigation Experiment was flown in a modified Spacehab rack on the Mir Spacehab STS-79 mission in August 1996.

The remainder of the paper is divided into two sections. The next section addresses the fundamentals of $\mu \mathrm{g}$ vibration isolation with a discussion of the relative merits of passive and active isolation approaches. The final section presents a description of a new vibration isolation system called g-LIMIT, which is designed for the ISS Microgravity Science Glovebox (MSG).

\section{FUNDAMENTALS OF MICROGRAVITY VIBRATION ISOLATION}

The basic objective of a vibration isolation system is to attenuate the accelerations transmitted to an isolated experiment either from a vibrating base or from directly applied disturbances generated by the experiment. Umbilicals, which pass resources such as power, data, and cooling fluids to an experiment, are the disturbance transmission path from the base to the isolated experiment. Direct inertial forces may result from crew contact or payload-generated sources such as pumps, fans, motors, and structural vibration of the isolated experiment. The transmissibility is defined as the magnitude of the transfer function from base acceleration to platform acceleration and is used to measure the attenuation of base motion as a function of the frequency. Whereas the mass of the isolated system and the umbilical stiffness and damping dictate the response characteristics of the system, these discrete elements are often selected for the purpose of shaping the dynamic response of a system to provide passive vibration isolation. This passive system behaves like a low-pass filter, transferring disturbances at frequencies below the damped natural frequency and attenuating disturbances at higher frequencies. Improved isolation from base motion is achieved by decreasing the break frequency and maximizing roll-off above the break frequency, where the slope of the roll-off depends on the damping (for an undamped system this slope is $-40 \mathrm{~dB} / \mathrm{dec}$ ade). Since it is typically not desirable to increase the payload mass, the break frequency may be reduced by designing the umbilicals 
to minimize stiffness. However, for small payload masses, achieving isolation at frequencies lower than one $\mathrm{Hz}$ by reducing stiffness is not possible with reasonable rattle-space constraints ( \pm one centimeter).

Another key deficiency associated with passive isolation systems is the inherent trade between resonance and high frequency attenuation. A resonant peak in the transmissibility function occurs at the natural frequency, the magnitude of which is determined by the damping. Greater damping results in more suppression of the resonant amplification, albeit at the expense of reduced attenuation at higher frequencies. Thus, when selecting the parameters of a passive isolation system a design trade must be made between resonant damping and high frequency attenuation.

Another deficiency of passive isolation is the inability to reject inertial disturbances. To improve upon attenuation of disturbance forces applied directly to the mass with a passive system, either the platform mass must increase or a stiff spring must connect the platform to the base (assuming the base is sufficiently massive). Since improved base motion isolation is achieved by softening the spring connection for a fixed mass, the objectives of base motion isolation and direct disturbance rejection are in opposition and cannot be simultaneously achieved. That is not the case with an actively controlled vibration isolation system.

\section{Active Control Concepts}

In order to provide a quiescent acceleration environment to an experiment, an active isolation system must sense and cancel the acceleration of the experiment. Typically a high-frequency acceleration feedback control loop is implemented to cancel the sensed accelerations and a low frequency position feedback control loop is used to center the platform in the sway space while following the quasi-steady motion of the vehicle. By sensing relative position and absolute acceleration of the platform the active control system forces the platform to follow the very-lowfrequency motion of the base while attenuating the base motion at higher frequencies. In essence, the isolation system must provide a soft suspension with respect to base motion disturbances, while providing a stiff suspension with respect to inertial (directly transmitted) disturbances. These competing objectives cannot be attained with passive isolation, but require active isolation with inertial acceleration feedback.

Comparing the passive system described above with a closed-loop active isolation system indicates that the acceleration, velocity, and relative position feedback gains may be viewed as effective mass, damping, and stiffness, respectively, and may be used to modify the dynamic response of the system. Hence, active control remedies the key deficiencies in passive isolation: direct disturbance rejection and the resonant peak/high frequency attenuation trade. Acceleration feedback is beneficial for attenuating direct disturbances by effectively increasing the dynamic mass of the isolated payload. By designing with frequency dependent gains, active control can effectively add damping in the break frequency region to attenuate the peak resonance without adversely affecting the attenuation at higher frequencies.

Two primary approaches are employed to provide active vibration isolation for microgravity payloads. ARIS has been baselined to isolate $50 \%$ of the U.S. allocation of ISPRs to be flown on ISS. Sub-rack level isolation systems such as g-LIMIT (described below). STABLE, and MIM provide a complementary approach to rack level isolation by providing vibration isolation directly at the rack to payload interface. The primary benefit of payload level isolation is the ability to implement higher bandwidth control laws since the payload structural frequencies tend to be significantly higher than the uncertain low frequency rack flexible modes. Additionally, the umbilicals required by an individual payload are substantially less than those of an entire rack, thus reducing the disturbance forces transmitted to the isolated payload. Rack isolation benefits from the economy of one isolation system for the entire rack as opposed to individual systems for each payload within the rack. Reference 5 presents a thorough description of the relative merits of passive, active rack and active payload level isolation systems with the highlights given in Table I. 
TABLE 1. Comparison of Isolation Approaches.

\begin{tabular}{|c|c|c|}
\hline Type & Advantages & Disadvantages \\
\hline Passive & $\begin{array}{l}\text { - Low Cust } \\
\text { - Low Manninance } \\
\text { - Reliable } \\
\text { - Vo Power }\end{array}$ & $\begin{array}{l}\text { - Isolate only high frequencies } \\
\text { - Large volume } \\
\text { - Cannot mitigate payload-induced } \\
\text { vibrations } \\
\text { - Resonance versus attenuation trade }\end{array}$ \\
\hline Active Rack & $\begin{array}{l}\text { - Low frequency attenuation } \\
\text { - } \quad \text { Mlultiple paylodas isolated with ona system } \\
\text { - } \quad \text { Standard payload interface }\end{array}$ & $\begin{array}{l}\text { - } \quad \text { Limited mitigation of payload } \\
\text { induced vibrations } \\
\text { - } \quad \text { Constrains payload dynamics } \\
\text { - } \quad \text { Highly sensitive to crew contact }\end{array}$ \\
\hline $\begin{array}{l}\text { Acrive Payload- } \\
\text { Level }\end{array}$ & $\begin{array}{l}\text { - } \quad \text { Low trequency attenuation } \\
\text { - } \quad \text { Mitigates payload induced vibrations } \\
\text { - } \quad \text { Optimized for individual payload } \\
\end{array}$ & $\begin{array}{l}\text { - Single payload per unit (more } \\
\text { resources) }\end{array}$ \\
\hline
\end{tabular}

\section{g-LIMIT}

A vibration isolation system named g-LIMIT (GLovebox Integrated Microgravity Isolation Technology) is being designed to provide active vibration isolation for $\mu \mathrm{g}$ science payloads using the Microgravity Science Glovebox (MSG). g-LIMIT is an evolution of technology demonstrated by STABLE, accomplished in part through a NASA HQ/Code UG Advanced Technology Development (ATD) Program project at NASA MSFC. g-LIMIT is scheduled for launch on the UF-1 mission and will be available to MSG investigators immediately after characterization testing.

g-LIMIT is an experiment level vibration isolation system with six degrees of freedom active control. g-LIMIT utilizes non-contact Lorentz force electromagnetic actuators, distributed digital signal processors, and a patentpending implicit relative position sensing design to provide a compact, state-of-the-art vibration isolation system for acceleration sensitive microgravity science experiments. Although g-LIMIT is a sub-rack level isolation system that can be used in a variety of applications, g-LIMIT is manifested for a MSG implementation. Standard MSG structural and umbilical interfaces will be used so that the isolation system is transparent to the user from an interface perspective. requiring no special accommodations. Figure 2 presents an assembly drawing of g-LIMIT.

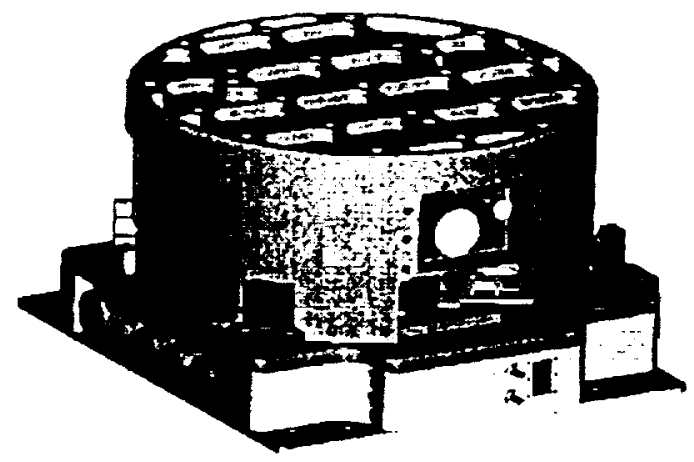

FIGURE 2. g-LIMIT System Assembly Drawing.

$\mathrm{g}$-LIMIT provides cancellation of inertial accelerations using a high gain, high bandwidth acceleration feedback control system that applies six independent forces to the isolated platform. A low frequency position feedback control system is used to maintain centering of the isolated platform. g-LIMIT is designed around three integrated isolator modules (IM), each of which is comprised of a dual axis actuator, two single axis accelerometers, two axes of implicit position sensing, control electronics, and an IR data-link across the actuator gap. This integration of sensing, actuation, and avionics into a single package is a key novel feature of g-LIMIT that allows for a more compact design as well as more general utility. The IM forms the basis of g-LIMIT and also provides the capability for an off-the-shelf kit for other isolation applications such as lockers, drawers, and other small volumes. Use of a 
co-located control law results in configuration independent software and negligible interfaces. Vibration isolation of larger masses is easily accomplished with g-LIMITT (or the IM kits) as well.

Another novel feature of $\mathrm{g}$-LMIIT is the patent-pending implicit position sensing technology which uses a drive coil to induce a signal on the actuation coil to sense motion much like a standard encoder. The g-LIMIIT system will not only provide a quiescent environment for MSG investigations, but it will also have the capability to generate pristine accelerations as specified by the isolated experiment developer. In this mode, a user-prescribed acceleration forcing function will be applied to the experiment while providing isolation from the ambient MSG acceleration environment. An additional capability will be the accelerometer-independent measurement of quasi-steady accelerations as a by-product of the isolation control system.

Demonstration of the required isolation performance in six degrees of freedom cannot be accomplished on the ground due to gravitational coupling, but requires testing in a $\mu \mathrm{g}$ environment. Long periods of experimentation are necessary to characterize the low-frequency behavior, which is the most critical frequency range for active vibration isolation. During flight investigation, various control designs will be tested to determine performance and robustness characteristics. A Dynamics Characterization Payload (DCP) will be urilized to characterize the direct disturbance rejection and robustness capabilities of the various control designs.

\section{Control System Design Philosophy}

The two key issues characterizing an active control system are stability and performance. Stability is the tendency for a system to return to equilibrium when disturbed. Performance is simply a measure of the degree to which stated objectives are achieved with the active control system. Stability and performance are in opposition such that the greater amount of performance one designs for, the lesser the amount of stability (margin) the system possesses. Robustness of the control system is also important since it is a measure of how much variation from nominal can be tolerated while preserving stability or performance.

One control design approach that emphasizes stability robustness over performance is "local control". In a "local control" implementation, the two axes of control in each IM are uncoupled by using co-located acceleration and position feedback to each axis of control actuation. Local control is known to possess good stability robustness when co-located acceleration or velocity is used for feedback. However, local control neglects the interaction between control channels and the dynamic coupling between axes, hence, emphasizing robust stability at the expense of performance. This is also an asset of local control in terms of implementation, interfaces, and utilization since the parameters are independent of the system properties to a great extent and need not be modified for different payload configurations. In local control, a fixed, configuration independent hardware/software design may be implemented. This feature results in the modularity and general utility of g-LIMIT.

Alternatively, "centralized" control will be implemented as well. "Central control" uses the distributed acceleration measurements to compute the rigid body accelerations in a platform-fixed coordinate system, computes control forces resolved at the origin of the platform coordinate system (typically the center of mass), and appropriately distributes the force commands to the actuators. Central control depends on knowledge of the system properties such as mass, inertia, and umbilical stiffness to determine the appropriate control forces and the correct force distribution among axes. Each axis may be treated independently, but the rigid body coupled motion of the system including the control force of each actuator is taken into account. It should be stated that central control designs can be more robust than local control designs, but the configuration of the actuators and sensors must be known to implement central control. Hence the software for central control is configuration specific.

A third control structure is "distributed control" which implements a central acceleration control law and local position control laws. Distributed control is the most efficient implementation from a computational perspective since the low-authority position control law computations are performed at the IM, thus reserving the PIP computational capacity for the higher performance acceleration control laws.

Implementing these general control system architectures enables the investigation of a wide range of control design methods and allows a thorough evaluation of the robustness and performance issues associated with microgravity vibration isolation. 


\section{REFERENCES}

1. Boeing Detense \& Space Group .Missiles and Space Division, "System Specitication tor the Internation.al Space Station," Specification \#41000D. Nov. 1. 1995.

2. Edherg. Don. Boucher. Rober. Schenck. David. Nurre. Gerald. Whonon, Wark, Kim, Young, and Alhom. Dean, "Results of the ST +BLE Mcrogratity Vibration Isolation Flight Experiment." presented at the 19th Annual A.AS Guidance and Control Conierence. Feb. 7-11. 1996. Breckenridge. CO.

3. Tryggvason. B. V., Stewart. B. Y., and DeCarufel. J.. "The Microgravity Vibration Isolation Mount: Development and Flight Test Results". I.AF Paper Vo. IAF-97-J.2.04. Presented at the $48^{\text {th }}$ International Astronautical Congress. Turin, Italy, October 6-10, 1997.

4. Bushnell, G. S.. and Becratt. M. D. ".Microgravity Performance Flight Characterization of an Intemational Space Station Active Rack Isolation Protolype System." in Proceedings of The I6th IEEE Instrumenation and Measurement Technolog: Conference (I.MTC199), Venice. Italy. May 24-26, 1999.

5. Grodsinsk?. Carlos .W. and Whorton. Wark S. "A Survey of Active Vibration Isolation Systems tor Microgravity Applications." Journal of Spacecraft and Rockets, submitted for publication. 